\title{
Filigrane
}

Écoutes psychothérapiques

\section{Le thérapeute, malade de son amour du métier}

\section{Marie Desrosiers}

Volume 17, numéro 1, printemps 2008

L’avenir du clinicien I

URI : https://id.erudit.org/iderudit/018793ar

DOI : https://doi.org/10.7202/018793ar

Aller au sommaire du numéro

Éditeur(s)

Revue Santé mentale au Québec

ISSN

1192-1412 (imprimé)

1911-4656 (numérique)

Découvrir la revue

Citer cet article

Desrosiers, M. (2008). Le thérapeute, malade de son amour du métier. Filigrane, 17(1), 121-141. https://doi.org/10.7202/018793ar

\section{Résumé de l'article}

L'auteur témoigne du travail de dégagement psychique d'un traumatisme lié à sa pratique de la psychothérapie psychanalytique. À partir d'événements liés au contexte de cette pratique, l'auteur illustre l'effort de décontamination auquel le thérapeute peut être confronté en tentant de composer avec différentes influences éprouvées comme externes à lui. Le cumul d'événements ayant cours dans la pratique, combiné à l'infiltration de la culture ambiante dans l'espace analytique sont d'abord mis à l'avant-plan pour situer la mise à l'épreuve qu'éprouve le thérapeute. Ce dernier est présenté dans la position de la personne traumatisée, cherchant à retrouver sa capacité à surplomber une scène, afin de remettre en mouvement la force vive de son investissement du métier et de rendre plus conciliable son désir d'analyse avec la réalité de la pratique. 


\title{
Le thérapeute, malade de son amour du métier
}

\author{
marie desrosiers
}

L'auteur témoigne du travail de dégagement psychique d'un traumatisme lié à sa pratique de la psychothérapie psychanalytique. À partir d'événements liés au contexte de cette pratique, l'auteur illustre l'effort de décontamination auquel le thérapeute peut être confronté en tentant de composer avec différentes influences éprouvées comme externes à lui. Le cumul d'événements ayant cours dans la pratique, combiné à l'infiltration de la culture ambiante dans l'espace analytique sont d'abord mis à l'avant-plan pour situer la mise à l'épreuve qu'éprouve le thérapeute. Ce dernier est présenté dans la position de la personne traumatisée, cherchant à retrouver sa capacité à surplomber une scène, afin de remettre en mouvement la force vive de son investissement du métier et de rendre plus conciliable son désir d'analyse avec la réalité de la pratique.

«Love is not a victory march, it's a cold, a very broken Halleluia... »

Leonard Cohen

\section{La pratique analytique et son potentiel traumatique}

$\mathrm{P}$ arler directement à partir de notre expérience personnelle de la pratique analytique suscite parfois un certain désir, un plaisir de communication, mais il peut aussi arriver que cela éveille une certaine répulsion, une hésitation, une réserve qui touchent à la honte, au sentiment d'exclusion ou à la défaite. D'autres raisons nous rendent parfois très discrets au sujet de ce que ce quotidien peut nous faire vivre, autant la pudeur, la difficulté à rendre compte que la nécessité de garder pour soi ses propres énigmes. C'est malgré tout ce que je me propose ici : parler de la pratique à partir de ma propre pratique. Au moment de cette écriture, deux voies m'encourageaient à poursuivre cette communication. D'abord, j'ai gardé de Granoff (2004), dans Le désir d'analyse, l'idée qu'il ne devrait pas être question de nous servir des erreurs ou des ratés dans la pratique de façon à en faire soit l'éloge, soit l'analyse complaisante ou repentante, mais plutôt de viser autre chose ${ }^{1}$... Puis, dans La démocratie en cruauté, Major (2003) me semble situer la difficulté de l'analyste à renoncer à son amour-propre comme une entrave à son devoir d'élaboration ${ }^{2}$. Mais plus encore, l'auteur y relance l'espérance d'un au-delà de la cruauté auquel la psychanalyse peut réellement contribuer et cela réveille une parcelle d'idéal que l'on ne devrait pas trop facilement céder.

Pour ma part, j'en suis venue à penser qu'il est grandement temps que les praticiens se penchent ensemble, dans notre culture qui permet encore que l'on 
puisse se parler, sur la blessure constante et répétitive qui est faite à notre potentiel de pensée et de parole, malgré l'éloge que l'on fait souvent de la liberté qui caractériserait notre chère démocratie. Peut-être devrons-nous davantage apprendre à rendre compte du trouble que nous occasionne la pratique analytique, non pas uniquement en dénonçant les attaques que nous subissons au plan individuel, groupal ou social mais surtout, tenter de comprendre ce que cette attaque suscite sur nos propres modes de penser et de pratiquer l'analyse ou la thérapie psychanalytique. Peut-être nous faudra-t-il analyser nos résistances à penser la chose sociale avec nos propres outils et prendre en compte les diverses tentations que nous éprouvons quand nous tentons de nous déprendre des influences culturelles dans lesquelles nous baignons, en particulier afin de rendre plus visible la puissance assourdissante et aveuglante d'un courant de fond qui fait son œuvre.

\section{Un cumul qui précède l'écriture}

Les événements qui se cumulent pour le thérapeute, dans la pratique du métier, sont nombreux et variés. Quand ces événements semblent avoir cours dans la situation assez bien délimitée entre le thérapeute et le patient, on suppose que des repères assez habituels viennent éveiller le thérapeute à des conflits et à des résistances qu'il s'attend à devoir situer du côté d'un infantile toujours actif au sein même de la situation analytique. Mais il arrive que des événements semblent davantage extérieurs, débordant la scène occupée par le patient et le thérapeute, et l'on pourrait sous-estimer leur effet d'infiltration à l'intérieur même du bureau. Pourtant, ce lieu n'est ni aseptisé ni à l'épreuve des ouvertures sur la culture ambiante puisque, en fait, ni l'un ni l'autre des protagonistes n'est à l'abri des influences externes. Ni, bien sûr, à l'abri des influences internes... mais comment discerner à coup sûr la source même d'une confusion ou d'une contamination ?

L'indécidable et la tentation de trancher entre des options, de façon à éliminer un dualisme trop dérangeant, apparaissent parfois comme les premiers signes d'une prise en compte d'un effet tirant vers le traumatique mais inaugurent déjà les possibilités de son surpassement par le renoncement à une trop rapide césure. Ces options se présenteront tout particulièrement de façon à ébranler le jugement qu'a le thérapeute sur la réalité : qu'est-ce qui vient de moi, qu'est-ce qui vient du monde ou de l'autre? De quoi est composé cet «autre» et quelles figures prend-il sur le plan des réalités, celles-là même qui sont placées au-dehors et qui se recouvrent de faits si flagrants qu'on osera à peine les questionner? Que reste-t-il au thérapeute pour qu'il remette en mouvement sa capacité à douter de «la réalité » et pour qu'il redonne force à ses propres mouvements internes de façon à se pencher sur ses propres entendements ou perceptions, de façon à relancer les auto-théorisations qui jusqu'ici lui avaient permis d'avoir une certaine saisie du monde et de lui-même et à réinventer du nouveau?

En-dehors de son bureau et sans la présence directe du patient, le thérapeute se voit confronté à des intrusions ou à toutes sortes d'interférences qui n'émanent pas exclusivement de sa propre personne et ces tiers se présentent parfois à lui comme 
porte-parole de la communauté plurielle : celle dans laquelle il se situe comme tout citoyen ainsi que la communauté plus restreinte de psychothérapeutes à laquelle il croit appartenir. Ainsi, ces représentants d'influences externes viennent ajouter des voies, de la cacophonie ou du bruit, à la représentation qu'il se fait de lui en rapport avec son travail. Un effort de décontamination semble particulièrement exigé du thérapeute dans certaines situations potentiellement traumatiques de sa pratique.

Partant d'événements qui se sont produits dans le cours de ma vie professionnelle sur une période assez brève, j'ai tenté d'extraire ce qui m'est apparu comme pouvant relever de cas de figures se retrouvant sous le couvert de différents scénarios que d'autres pourraient aussi rencontrer. Pour le thérapeute, les défis concernent autant la mise à l'épreuve de sa capacité de penser que celle d'être en lien et de se maintenir dans ce lien avec ce qui, auparavant, lui avait fait opter pour ce métier. C'est dans ce sens que nous annoncions dès le départ, en titre de ce texte, que l'amour du thérapeute pour le métier, en particulier son attachement à la psychanalyse, risquent d'être mis à mal de diverses façons. Le poète du début, quant à lui, constatait qu'il serait vain de croire à une marche victorieuse de l'amour puisque celui-ci prend aussi le visage d'une chose froide et brisée, «a broken halleluia». Ce texte ne propose pas de solutions, ni à la douleur des liens brisés, ni à l'éprouvé des défaites du narcissisme, ni à l'équilibre toujours vacillant de la psychanalyse quand elle cherche à occuper une place auprès des autres domaines concitoyens qu'elle a peine à reconnaître comme co-sanguins. Ce texte cherche simplement à illustrer quelques voies d'exploration qui furent empruntées pour tenter de transformer un effet traumatique global, aussi intense qu'indéterminé, en quelque chose de plus conciliable avec le désir de poursuivre dans la voie de la pratique analytique.

\section{L'Événement mis en récit}

À ce qui me revient rapidement à la mémoire, sans compter tout ce qui y fut oublié, j'avais reçu dans la même quinzaine le résultat de quelques expertises faites sur des patientes que je suivais depuis déjà un certain temps, en bureau privé. Celles-ci m'avaient toutes été référées en tant que victimes. Soit elles avaient vécu un inceste paternel précoce et une violence familiale sévère aux tous premiers temps de leur vie, soit elles avaient été victimes d'accidents de la route dont elles n'arrivaient pas à se remettre. Toutes disaient qu'on les avaient poussées à consulter, que la loi québécoise était ainsi faite et elles venaient d'apprendre leur droit d'obtenir réparation pour les dommages qu'elles avaient subi. Aucune n'arrivait à dire dans son propre langage de quels dommages il était question mais, après quelques rencontres, toutes allaient apprécier le fait de venir parler à quelqu'un qui ne leur demandait pas à l'avance de justifier de long en large leur présence. Elles pouvaient donc venir parler grâce à une reconnaissance sociale de leur statut de victime mais aussi indépendamment de ce statut. Dans le cours de leur thérapie, j'avais eu accès aux expertises parce qu'elles-mêmes en 
avaient reçu une copie avec laquelle, d'ailleurs, elles ne savaient pas quoi faire. Avant de se livrer aux expertises, toutes m'en avaient voulu de les laisser aller seules, vers ce qu'elles qualifiaient «d'abattoir». Selon leurs dires, lors de ces "interrogatoires", toutes étaient restées perplexes devant les mêmes questions: qu'est-ce que le thérapeute fait avec vous et quels sont ses objectifs? quels sont vos buts à court, à moyen, à long terme? L'une d'elles n'avait que répondu: "mes objectifs de vie?... je n'en sais rien... survivre?».

Une autre à qui on avait demandé s'il lui était arrivé de penser à la mort, en fantasme, avait hésité à répondre en se rappelant tout le temps qu'elle avait dî mettre afin d'établir une différence entre la "réalité » et autre chose qui existait parfois dans sa tête. Dans le rapport, l'expert avait répondu à son effort d'honnêteté en précisant qu'il vaudrait mieux signaler cette femme à la protection de la jeunesse. Il y mettait à l'avant-plan son souci «si la thérapeute ne collaborait pas... », anticipant certaines réactions chez la patiente quand tomberait l'annonce de la cessation de la thérapie. Révélant à la patiente qu'il n'y avait plus d'avancée possible dans une thérapie, que cela était "plafonné», on craignait qu'elle garde une dépendance et que la thérapeute ne réussisse pas à l'en déprendre pendant les semaines qui suivraient. L'expert laissait une ouverture, en cas de rechute, en recommandant (statistiques à l'appui) le recours à une thérapie dialectique-comportementale qui avait fait ses preuves et qui aurait déjà dû lui être recommandée. Or, chez ce tiers-payeur, il semblait que toute expertise ait valeur de vérité absolue. C'est quelques temps après cet événement, qui avait été éprouvé comme un désaveu de notre travail, qu'une première demande d'enquête au sujet de ma compétence professionnelle, en lien avec cette thérapie, fut déposée.

Une autre patiente était entrée en crise sévère à la suite de l'expertise où elle leur avait "tout déboulé ce qu'ils insistaient pour savoir», mais sans rien en ressentir. Puis, elle était venue s'effondrer dans le bureau quand cette scène récente était parvenue à son point de dégel, ne pouvant que dire en après-coup: "l'expertise, c'était pire que les viols parce que les viols, ça durait juste quelques minutes». Déplacement de scènes qui seraient réapparues par d'autres voies, pourrait-on dire, mais où l'on finit tout de même par se demander, dans la situation actuelle: était-ce vraiment nécessaire? Elle était revenue quelques semaines après, s'étant soumise à la recommandation de l'expertise de recevoir une médication et, s'adressant à moi: "je le fais pour vous, pour vous garder, je dois leur obéir...». Puis, elle était revenue la semaine suivante: "Écoutez-moi, disait-elle en me pointant, ils veulent faire disparaître mes crises, comprenezvous? Vous, vous devez me croire: j'en ai besoin, de ces crises ». Ce message, on l'aura deviné, ne s'adressait pas qu'aux prescripteurs de médications dont elle craignait l'efficacité. Elle m'avait expliqué la terreur que lui inspirait l'idée d'être "stabilisée», elle serait perdue, peut-être même allait-elle recommencer à faire des trous dans le mur, saurait-elle comment s'y prendre pour ne pas frapper les enfants? "J'ai recommencé la cocaïne, jeta-t-elle finalement, sinon «leurs» médicaments me rendent trop amorphe et j'arrive pas à prendre soin des petits 
comme avant...». Belle justification? compromis d'adaptation sur-déterminé par ce qui lui avait été si familier? Sa confiance s'effritait, comme si on venait lui confirmer le «faux»du «c'est pour ton bien » dans lequel elle avait vécu, depuis toujours. Un peu plus tard, on allait nous indiquer que la toxicomanie ne pouvait pas faire partie de ce traitement parce que cela ne se rattachait pas aux violences sexuelles et physiques subies dans l'enfance.

Une autre, encore, pas plus révoltée que les précédentes mais agissant de façon plus bruyante sur la scène externe, avait entamé des procédures judiciaires pour exiger de poursuivre sa thérapie, c'est-à-dire que l'organisme continue d'assumer les frais d'une thérapie dont elle disait avoir besoin. Plus que juste la réalité d'une insuffisance d'argent combinée à la réduction de sa capacité physique limitant l'accès à un emploi, elle exigeait d'abord et avant tout qu'on remplisse une promesse qui lui semblait avoir été portée par le message et les incitations des institutions sociales. Dans cette même quinzaine de réception d'expertises, je me retrouvais donc, curieusement, avec un jugement de cour qui «forçait» le retour d'une patiente en thérapie... ce qu'elle ne fit pas, sa victoire ayant sans doute été déjà consommée à partir du jugement favorable.

Durant cette période, un échange imprévu et anodin m'avait laissée sans voix après qu'un des psychiatres d'une patiente soit entré en contact avec moi alors qu'elle venait de se faire ré-hospitaliser. Ayant révisé le diagnostic de ses confrères et croyant que cela m'aiderait à mieux travailler, on me prévenait: "savezvous qu'il existe des thérapies qui induisent des faux souvenirs, le savez-vous?", "mais aussi, j'imagine que vous savez ce que c'est qu'un trouble factice? ». Peutêtre était-ce la passion du ton qui m'avait troublée ou encore, peut-être était-ce l'agglutination des effractions externes qui commençait à se faire sentir. Sur le coup, je me rappelai que nous n'en étions pas au premier diagnostic, la patiente m'en avait dressé la liste et ceux-ci variaient au gré des changements de lieux des traitements. Je me rappelais d'ailleurs m'être questionnée, en lien avec cette patiente, sur l'impact qu'aurait la centralisation éventuelle des données médicales (en devenant informatisées et accessibles) sur le besoin qu'ont certaines personnes de s'éparpiller et de morceler les informations à leur sujet. J'en venais même à penser que le clivage pourrait être conçu comme un droit ou, au moins, considéré comme une réalité dont le sens transcende les exigences administratives. Peut-être que j'en arrivais ainsi moi-même à commencer à emprunter à la logique des droits qui implique une certaine conception de la loi.

À cette époque, le désir de parler de la pratique, surtout de son potentiel traumatique pour le thérapeute, cela m'apparaissait puis disparaissait constamment, davantage comme une tentation qu'un désir bien affirmé. J'allais de vagues en ressac. Peut-être la réelle brisure s'était-elle faite sentir lorsque j'avais dû encaisser une deuxième demande d'enquête pour vérifier ma conformité à la pratique de la psychothérapie. Le processus d'enquête est généralement ce qui va déterminer si une plainte devrait être déposée en bonne et due forme auprès de l'ordre professionnel auquel j'appartiens. En soi, on peut ici penser à une mesure 
de routine à laquelle tout professionnel peut être soumis, indépendamment du bien-fondé ou non de la demande, indépendamment de l'issue de ces procédures. Dans la réalité, on peut imaginer que ces demandes récurrentes puissent provoquer un dérangement. Elles viennent heurter notre attachement au caractère si privé de ce qui se joue dans notre bureau. Il me semblait que le temps pour l'écriture m'était définitivement dérobé puisqu'il me fallut m'expliquer à répétition, justifier ma pratique professionnelle à des gens d'une autre langue. Une tâche habituellement secondaire de mon travail prenait ainsi le premier plan puisqu'il me fallait alors répondre à des questions, comme l'étranger doit justifier sa seule présence, avant même de faire comprendre sa propre différence.

\section{Ne pas se savoir étranger}

Comment avais-je pu paraître si étrange à d'autres ? C'est peut-être là, au cœur de cette interrogation, qu'il m'aura fallu réapprendre les risques que quiconque court à ne pas se savoir étranger. J'allais devoir admettre avec plus de conviction qu'il y a des risques à ne pas vouloir se reconnaître en tant qu'étranger. Mais quel était, d'ailleurs, ce «défaut» qu'on sembla souligner en s'adressant à moi, était-il vraiment lié à une étrangeté dans mon discours ou cela se logeait-il dans ma façon de faire qu'on suspectait d'être non- conforme? Le fait est que, s'expliquer nous coûte toujours quelque chose, si ce n'est le temps qu'on doit y mettre, mais il est heureusement possible qu'on en retire aussi des dividendes. Si rendre compte est déjà compliqué lorsqu'on traduit à un tiers-ami notre pratique de psychothérapie, rendre des comptes peut s'avérer un défi de taille si la confusion des langues occupe l'avant de la scène dans ce rendez-vous.

Dans toute situation d'enquête, on sait bien que le regard d'autrui vient parfois mettre en relief certains doutes qu'on a soi-même à notre sujet, bien que ceux-ci n'appartiennent pas nécessairement à la catégorie des doutes sur notre intégrité. Par exemple, dans certains parcours plus difficiles, il arrive qu'on finisse soi-même par se demander: «et si je faisais plus de mal que de bien?». Ou encore, on va parfois jusqu'à imaginer un substitut à notre propre personne, à défaut de ne pouvoir s'enlever du décors lors de rudes mises à l'épreuve cliniques ou à défaut, tout simplement, d'élaborer la haine dans le contre-transfert. Malgré notre conviction préalable d'une indication de thérapie analytique, il arrive qu'on finisse tout de même par penser: «et si je m'étais leurré sur la demande de cette personne-là?», ce qui reviendrait parfois à dire «et si tout cela n'était que mon propre désir?».

Ces questions, posées dans l'intimité de notre pensée ou avec les «nôtres», peuvent prendre une nouvelle allure quand il nous faut en discourir avec des gens d'une autre langue. Dans un tel contexte, ces mêmes questions risquent de déferler, faisant retour à une vitesse vertigineuse avant que le thérapeute réussisse à regagner une certaine stabilité. Ces questions concernant, par exemple, le consentement éclairé d'un sujet à entreprendre une thérapie, les procédures concrètes établissant ce consentement, les limites de l'intervention et l'indication d'une 
approche plutôt qu'une autre, les aspects légaux pour légitimer telle ou telle dimension du cadre, nous nous les posons le plus souvent en regard d'un contexte clinique particulier. Rarement, nous les élaborons en-dehors du sens qu'elles prennent dans tel ou tel suivi. De plus, l'expérience clinique nous permet de développer une saine capacité à ne pas tout savoir à l'avance et cela permet même d'offrir au patient un objet relativement malléable qu'il pourra utiliser pour le jeu de la thérapie. Mais sur la scène extérieure où le thérapeute exerce son métier, la fermeté de ses convictions sera convoquée d'une autre façon et son tact ainsi que son aptitude à la traduction devront se mettre au service de sa propre survie.

On peut concevoir que l'effet cumulatif dont il est question ici vient en quelque sorte placer le thérapeute dans la position de patient traumatisé mais il est encore plus juste de penser cette position en termes de potentiel traumatique où le vacillement guette le thérapeute qui cherche sans cesse un nouvel ancrage. L'effort du thérapeute, afin de reconnaître de multiples effractions et de symboliser quelque chose quant à l'éprouvé d'une quantité excessive, vient forcer notamment l'auto-analyse, en supposant que celle-ci reste possible. C'est ainsi que, plus que jamais peut-être, le thérapeute devra lui aussi déconstruire les scénarios, examiner la valence répétitive dans ce que représentent ces événements, les reprendre morceaux par morceaux et voir ce qui en a permis l'assemblage au-dedans de lui, tout cela afin de se réapproprier la force vive permettant son investissement du métier.

\section{Sur fond d'hyper-réalité}

Pour ajouter une autre perspective à ce tableau plutôt banal, ce climat de suspicion faisait suite, dans ma pratique personnelle, à un non moins banal moment d'accalmie de clientèle. Encore ici, rien de spécial, puisque la plupart des thérapeutes traversent probablement de façon récurrente dans le cours de leur carrière, des moments de doutes et d'angoisses... et ils rêvent toujours que ces moments ne seront que des transitions et qu'ils ne viennent pas annoncer la fin. Mais dans les creux de vagues, il reste tout de même possible pour eux de s'imaginer devant la fin autant que la faim. «La psychanalyse ne me fait plus vivre » est un discours qui remontait à mes oreilles, commençant à se détacher avec clarté du bruit ambiant. Mais que veut dire, au fond «vivre de la psychanalyse » et comment l'entendre en particulier quand un mode particulier d'écoute et de travail nous procure l'accès autant à une source irremplaçable de satisfactions

qu'à une source unique de revenus ? Évidemment, parmi les discours qui rabattent la possibilité d'en penser réellement quelque chose, il y a celui dénonçant la persévérance à vouloir maintenir une orientation (n'exercer que la psychothérapie psychanalytique ou l'analyse en bureau privé, comme seul métier) ce qui est parfois vu comme de l'acharnement, voire de l'idéalisme entêté. Un discours condescendant vient pointer les regrets qu'on se sentirait parfois coupable de ne pas avoir. Néanmoins, la pression reste forte à exercer un «métier convenable».

L'accalmie de cette saison-là ne semblait pas venir uniquement du fait que le téléphone ne sonnait plus puisqu'il lui arrivait encore de sonner. J'avais plutôt 
l'impression que même ce qui réussissait à se nouer dans mon bureau se détissait parfois plus facilement qu'avant, laissant alors des trous dans mon horaire que je n'avais jamais vus jusqu'ici.

Adoptant moi-même une position clinique plus explicite, en particulier face aux thérapies destinées à des personnes se présentant comme victimes et rattachées à des tiers-payeurs, le refus de ces tiers m'apparaissait lui aussi plus explicite. "On ne veut pas ce genre de thérapie-là», m'avait-on dit au cours de cette même période. La limite énoncée par ce "on ne veut pas... », cela s'arrimait assez bien, par ailleurs, à ce qui prenait forme en moi, c'est-à-dire au désir de renoncer plus définitivement à un type de cadre de travail particulier. La clientèle, quant à elle, s'était présentée comme déjà prise dans un système de soins et chacune de ces victimes déclarées m'avait permis au cours des ans d'apprendre à entendre l'écho des traumatismes, les marques du désaveu, les traces de l'effacement et de l'éradication.

Ce refus de traitement de la part d'un tiers payeur était survenu à propos d'une pré-adolescente qui se présentait avec divers événements qui s'étaient cumulés pour elle dans les dernières années. Malgré son jeune âge, la jeune fille avait déjà fait plusieurs thérapies et elle semblait maîtriser parfaitement un discours psychosocio-politique sur les victimes, leurs droits et leurs malheurs et elle réussissait à m'expliquer les effets de l'inceste sur le développement des enfants et les stratégies adaptatives pour se sortir de "ça». C'est peut-être en raison même de l'étalement de ses grandes «connaissances», de son attitude professorale sur les méfaits de la violence, mais aussi en réponse à cette maîtrise apparente dans un discours désincarné, que je lui avais offert tout simplement de me parler d'elle. Elle avait paru ravie de recevoir cette invitation à parler, selon elle "d'autre chose que des abus sexuels» et se demandait si on en avait le droit. Une approche éducative avait déjà été expérimentée dans les autres thérapies et je proposais de poursuivre dans une autre voie. C'est dans ce contexte qu'on finit par m'informer que cette approche ne coüncidait pas avec la philosophie d'aide aux victimes et qu'un autre psy serait mandaté pour offrir une aide plus adéquate. La petite cliente n'avait-elle pas eu raison quand elle demandait, sans une certaine inquiétude, si nous avions le «droit de juste parler»?

C'est à répétition que le thérapeute se retrouve au cœur d'une confusion où le patient se trouve face à un discours dont il ne saisit pas toujours la logique, comme si adultes et enfants cherchaient sans cesse une langue pour se rencontrer, sans y parvenir. Être l'interprète de qui ou de quoi ? finit-on par se demander... quand il s'agit de délimiter l'espace potentiel devant permettre la rencontre thérapeutique.

\section{Les ententes modèles et les modèles de traitements}

D'un point de vue strictement rationnel, on peut toujours recouvrir de réassurances superficielles notre inconfort et se dire «c'est mieux comme cela, pour cette jeune fille, et c'est mieux pour moi parce qu'il y a d'autres cas où le contexte est moins compliqué ». Le mieux serait pensé ici par rapport à une entente 
modèle qui ne s'établirait pas à l'avance sur un litige qui risquerait très tôt de mener à la cessation brutale d'un lien. Or, même dans les analyses les plus «consenties», ne le sont-elles pas toujours sur fond de litige, d'imprécision, de conflit potentiel?

Réal Laperrière (1999) avait offert, il y a déjà quelques années, une riche illustration d'un trouble qui se manifeste chez le thérapeute analytique et auquel il avait donné le nom de malaise de l'imposteur. Même si cette illustration prenait comme toile de fond la pratique institutionnelle en pédopsychiatrie, l'auteur y questionnait un malaise beaucoup plus généralisé qui serait le produit de la situation analytique et il démontre comment ce malaise est intrinsèquement lié au travail de l'analyse. Nous suivons l'auteur qui place la question du vrai comme surgissant de l'imposture et qui situe le malaise à la source même d'un travail analytique authentique. Dans cet exemple clinique, les questions soulevées rejoignent notre propos: le thérapeute se demande s'il est entrain d'œuvrer clandestinement à créer un espace qui, par ailleurs, ne répond pas à ce qui serait demandé au couple patient-thérapeute. L'issue de la vignette en question a la particularité de suggérer des conditions qui auraient permis à l'auteur de garder son malaise pour lui, tout en continuant à se demander, en catimini, s'il est vraiment intègre dans son travail. Il en vient à se pencher sur le contexte de la pratique auquel on serait tenté d'attribuer la mise en place du trouble de l'imposture mais finalement, il dit être davantage interpellé par ce qui ferait écho à son histoire personnelle.

Pour reprendre le fil de la présente réflexion sur le potentiel traumatique de la pratique pour le thérapeute, deux éléments du travail de l'auteur précédent attirent notre attention. D'abord, concernant cet écho personnel auquel nous sommes renvoyés dans l'analyse du contre-transfert, celui-ci ne vient-il pas aussi se démarquer d'un bruit de fond beaucoup plus vaste qui rappellerait l'écho d'une compulsion collective et groupale de répétition à laquelle il peut devenir difficile de s'arracher? Nous y reviendrons puisque le climat ambiant dans lequel s'inscrit la pratique actuelle de toute psychothérapie analytique, institutionnelle ou pas, vaut la peine qu'on s'y arrête un peu.

Ensuite, que peut-on imaginer du thérapeute qui s'investit précisément avec des personnes sévèrement traumatisées et qui reçoivent un aval social par la reconnaissance du statut de victime, si l'issue ne se présente pas de façon aussi favorable que dans le texte évoqué plus haut? On peut d'abord se demander comment viendrait jouer la pression qui se fera sentir à traiter une victime plutôt qu'un sujet. Si le thérapeute choisit de ne pas se restreindre à une vision compassionnelle, comment sera accueillie sa façon de faire par ceux qui se sentent déjà rassurés dans leur conscience, du fait d'avoir permis le repérage de la victime et son assignation à un traitement? La question de la clandestinité de notre travail ne place-t-elle pas le thérapeute dans une position particulièrement inconfortable à certains moments où ce travail «tourne mal» ou est déclaré de l'extérieur comme «tournant mal»? Comment traiterait-t-il, le thérapeute, cette récurrence à devoir défendre son 
intégrité, à justifier sa différence et sa prétendue imposture quand l'industrie du traumatisme, pour emprunter l'expression aux deux auteurs suivants, se nourrit de son corollaire: l'exigence de bien-être, pour la victime (Eliacheff et Soulez Larivière, 2007,45).

Dans l'exemple de la jeune fille de tantôt, il s'agissait de présenter un fait divers courant, un refus ayant l'avantage d'être clair et s'exprimant sur le mode «on ne veut pas de...». La rupture subséquente survenait lors d'un moment préliminaire, avant que des éléments transférentiels plus marqués entre les deux protagonistes ne se déploient comme c'est le cas en cours de route. Mais lorsque les désengagements ou les ruptures, les vécus d'invasion extérieure ou les désinvestissements personnels se produisent à l'apogée d'une névrose ou d'une psychose de transfert, on peut imaginer que le thérapeute ne pourra pas se contenter d'une rationalisation à propos de la disqualification dont il se sent l'objet. De façon beaucoup plus affective, il risque d'éprouver une double souffrance, on pourrait dire une double peine d'amour qui le renvoie à une attaque contre ce qu'il porte en lui mais aussi à une rupture de lien avec un autre, au deuil d'une rencontre qui n'aura plus lieu. S'il se trouve le moindrement déstabilisé en lui-même, lui aussi court le risque de se poser alors... comme une victime!

\section{Sur fond d'intolérance}

Les auteurs du livre Le temps des victimes (Eliacheff et Soulez Larivière, op. cit.) conjuguent leurs expériences respectives en psychanalyse et en droit, ces deux domaines se voyant particulièrement interpellés par les demandes de ce qu'ils appellent l'industrie du traumatisme. Ils se posent en critiques et offrent une perspective historique de la notion de victime, de la place sociale que celle-ci occupe en remplacement de la place du héros et ils dénoncent certains impacts individuels et sociaux d'une prévalence du mode compassionnel mis au service d'un prétendu idéal de justice et de démocratie. Ils posent aussi leur critique d'un marché victimaire qui s'appuie sur une certaine conception du préjudice en faisant ressortir au passage comment, dans les années quatre-vingt, les concepts issus de la psychanalyse s'estompent puis s'effacent plus radicalement des manuels diagnostiques et des approches cliniques au moment même où des considérations politiques et économiques forcent la réinvention de la névrose de guerre. C'est ainsi, notent-ils, que celle-ci se voit remplacée d'abord par le «Post-Vietnam Syndrom» qui viendra se fondre dans le très populaire «Post-Traumatic Stress Disorder». Au fil du temps, dans la culture des soins tout comme dans la pensée populaire, le termes «victime et traumatisé» se voient de plus en plus confondus. Les auteurs font remarquer une nouvelle conception qu'ils énoncent ainsi : «La «bonne» victime n'a pas d'inconscient: elle ne jouit pas, elle n'est pas ambivalente, elle ne rationalise pas, elle met tout en œuvre pour être «reconnue», indemnisée et pour que l'auteur de son traumatisme soit puni afin qu'elle puisse «faire son deuil» et se reconstruire ». Les auteurs affirment enfin: "Ces victimes-là n'existent pas et n'existeront jamais, mais qui va le reconnaître?» (Eliacheff et Soulez Larivière, 
2007, 49). Comment imaginer que le thérapeute momentanément troublé par un excès de contamination avec la rencontre de la scène sociale puisse lui-même être mis à l'abri de la tentation vers ce glissement victimaire ? Peut-il s'en prémunir?

De façon générale au cours de cette période, il m'apparaissait que la demande ne semblait plus coïncider avec ce que j'avais à offrir. Peu à peu, j'en venais à me sentir différente et marginale, peut-être même déficiente. Plus que jamais, j'avais ainsi l'impression de nager en pleine confusion des langues, et cela avec l'éventuel patient, même quand aucun tiers ne faisait directement interférence. Peut-être était-ce moi qui n'avais plus la patience? Pressée par le désir de travailler, par l'anticipation du plaisir à faire mon métier, il m'arrivait de voir l'autre comme celui qui ne fait pas la bonne demande, mon impatience se confondant avec ce noyau d'intolérance que l'on porte tous en chacun de nous. Parfois, même lorsqu'un éventuel demandeur de thérapie analytique semblait venir s'inscrire dans une démarche «plus en profondeur» ou qualifiée par le futur patient avec ce type de vocabulaire un peu flou, il devenait notable que le monde externe finissait malgré tout par s'en mêler. Encore là, rien d'exceptionnel à la pratique quotidienne de tout thérapeute, sauf que je croyais observer un nouveau rapport de force se jouant avec les influences externes, plus virulentes et indiscutables, et j'arrivais beaucoup plus difficilement à les contrecarrer par les possibilités qu'offre la parole. La parole pouvait-elle encore faire le poids?

On a beau se dire «ce patient ne voulait pas vraiment, sinon il n'obéirait pas ainsi à son médecin, à son assureur»; on a beau invoquer la résistance individuelle, le manque de courage, la paresse quand on ne sait plus quoi penser d'autre sur le retrait prématuré dans un suivi qui semblait bien s'amorcer; on a beau avoir bien intégré cette idée chère à Ferenczi selon laquelle il ne nous resterait qu'à nous interroger sur nous-même, on a beau croire qu'on est de ceux qui savent dans quel monde on vit, il n'en demeure pas moins qu'à la longue, le thérapeute encaisse, à répétition, un contre-investissement.

Peu à peu, j'ai eu l'impression d'assister en direct à un changement de scène. De spectateur du sort que la psychanalyse subirait depuis ses tous débuts, de commentateur d'un thème qui fait couler l'encre dans toute revue psychanalytique qui se veut à l'heure du jour, je me suis à peine vue traverser le mur entre cette fiction d'un discours collectif et une autre réalité traumatique: la mienne. C'était devenu trop. Mais surtout, quelque chose était devenu indissociable. Je ne sais pas jusqu'à quel point, pendant des mois, il m'aura réellement été possible ou non de percevoir ce qui séparerait une scène sociale et externe où se joue à répétition l'effort de survie de la psychanalyse et discerner cette scène de ma propre position individuelle et interne.

\section{Le père, l'enfant et la psychanalyse}

C'est dans une confusion des scènes que peut survenir, il me semble, un trouble qui concernerait à la fois notre possible sentiment d'imposture mais plus que cela, qui risquerait de venir brouiller nos identifications les plus profondes. L'attaque à 
l'identité exige un effort de discernement des plus soutenus. Le thérapeute peut finir par se demander s'il est la cible d'une attaque contre la psychanalyse qui passerait à travers lui. Il peut aussi questionner ses difficultés individuelles à concilier son amour de la psychanalyse avec certains renoncements devant lesquels il est placé, face à l'exigence de gagner sa vie. Le départage entre les renoncements qui seraient ou non vraiment nécessaires pose alors un défi à son jugement.

Nous posons que le rapport du thérapeute à sa pratique peut se présenter avec sur-détermination dans le contexte de l'ébranlement potentiellement traumatique. Il pourrait avoir à se demander comment échapper à une identification à l'enfant s'il se sent rattaché à des fantasmes toujours en voie de résurgence qui risqueraient de le confondre avec la «chose» elle-même. Autour de différentes métaphores impliquant l'enfant de la psychanalyse, le thérapeute pourra se sentir glisser vers certaines positions, par exemple : «On tue un enfant»; «Un enfant est battu». On peut dès lors l'imaginer, le thérapeute, se prendre dans les pièges aberrants mais pourtant probables où un objet d'identification se verrait confondu à lui-même. Cela éveillerait dans son univers psychique des substitutions impliquant, par exemple, des fantasmes relatifs à «On tue la psychanalyse»; ou encore «La psychanalyse est battue, malmenée...». Ébranlé de l'intérieur, il pourrait s'éprouver comme si on le laissait tomber et peut-être même en venir à se vivre plus profondément comme l'enfant mal accueilli d'un célèbre article de Ferenczi (1929), ce qui nous ferait évoquer «Le thérapeute mal accueilli et sa pulsion de mort...».

On se retrouverait ainsi dans un registre où quelque chose qui dépasse et englobe la personne propre s'éprouverait comme menacée de ne pas advenir, de ne pas se maintenir en vie, d'être malmenée ou mise à mal. Que le conflit prenant une saveur de trahison se vive au sein même du monde psychanalytique ou qu'il se situe en rapport avec d'autres milieux, il risque, pour reprendre Pontalis ${ }^{3}$, de se vivre aussi sous ce mode passionné: «ma mère contre la tienne». Ma théorie contre la tienne; ma conception de la «chose thérapeutique» contre la tienne... cet engrenage bouscule toujours les fondements de chacun, parfois plus profondément enracinés qu'il n'y paraît, même dans les discours les plus aseptisés.

Tenant compte de ces enjeux si intimement privés, on peut se demander ce que la violence du choc répété avec des limites imposées à la pratique dans la réalité vient faire éprouver au thérapeute. La mise en doute récurrente à son sujet peut venir ébranler jusqu'à sa propre conviction à l'égard de son aptitude à douter: subitement, le thérapeute risque de devenir plus que convaincu. Ultra-convaincu. C'est peut-être rendu là qu'il n'aura plus le choix de questionner les sources mêmes de sa propre tendance au fanatisme.

\section{L'investissement : une condition au traumatisme}

Le trauma semble procéder par la combinaison d'une agglutination et il conduit le sujet, le thérapeute étant ici ce sujet qui nous préoccupe, à une certaine désorientation qui lui rend difficile la tâche de répondre à «qu'est-ce qui m'arrive ?», 
«pourquoi ça me tombe dessus?». L'amalgame de la puissance du déni et d'une force de répétition entraînent une position d'indécidable quant au lieu de la réalité. Dans le récit qui précède, l'agglutination se démontre dans l'énoncé des événements-mis-bout-à-bout mais peut-être, surtout, dans ce qu'on constatera qui n'est pas dit, dans ce qui y est devenu presque absent, atténué, effacé momentanément. Disparus, ces moments de grâce qui nous font connaître des moments de pure beauté au sein d'une séance que l'on perçoit comme une œuvre d'art. Éloignés, ces moments où l'on ressent l'émotion à découvrir, comme pour la première fois, l'effet magique d'une parole qui reprend place dans l'ordre des choses. Effacés, ces instants de bonheur à lire un auteur qu'on reconnaît comme proche parent et qui vient supporter ou ébranler avantageusement notre pensée. Annulées, ces connivences avec des collègues qui nous envoient précieusement un patient pour nous témoigner leur confiance. Même les motifs qui rendent à un certain moment plus qu'à d'autres le besoin d'argent ou de reconnaissance, le désir de liens ou de preuves d'appartenance, ces déterminants finissent par se dissiper dans le discours et se diluer dans l'événement, si bien qu'un peu plus et on croirait le thérapeute uniquement victime de sa pratique de la thérapie analytique et des interférences externes.

Vient un moment où, dans la tête du thérapeute qui serait en voie de basculer, risque de se produire, sous le mode de l'étrangeté, un semblant d'hallucination qui met en scène une attaque incessante. Piégé par une auto-conviction trouble mais débordante, le thérapeute se voit menacé, coup sur coup : quelque chose disparaît, s'absente, quelqu'un ou quelque chose est en voie de disparaître "pour vrai». Lui-même craint de disparaître ou encore, il craint que ce ne soit «la chose» (la psychanalyse ?) qui disparaisse... Sur le coup, il ne lui arriverait même pas de penser avec cette distance, voire cette sagesse, qui fait dire à Michel Gribinski, s'exprimant sur le sort qu'on fait subir à la théorie psychanalytique, qu'on s'occupe à partir du dehors comme du dedans «de la réviser avec tant de constance, tant de virulence parfois, qu'on a le sentiment qu'elle finit par jouer un tour à qui l'attaque, en lui devenant nécessaire» (Gribinski, 2007,10). Parfois à son insu, le thérapeute est déjà entrain de s'engager «au front», se sent «sur la première ligne», dans un état de «légitime défense »... alors qu' au fond, ne serait-il pas plus souhaitable qu'il accède simplement à une position de «résistance délibérée » qui lui permettrait davantage de penser par-devers lui, sans pour autant nier une réalité actuelle. Tout absorbé par une lutte, croyant sa perception guidée par une attaque, le thérapeute peine à reconnaître la force qui viendrait décupler les sentiments d'amour en jeu.

Dans un texte au titre évocateur L'amour des certitudes, Miguel De Azambuja (2004) nous met en garde contre la visée totalitaire de l'amour quand celui-ci ne se fonde que sur l'expérience exaltante de la poussée animiste. Il nous prévient que «la certitude de l'amour de l'objet éloigne le péril mélancolique» mais que «néanmoins, loger un objet à la place des certitudes n'est pas sans risques, car celui-ci peut devenir immuable...». Enfin, l'auteur rappelle que pour qu'une 
histoire d'amour parvienne à se dérouler, le sujet aura à «déloger l'objet de cette place (de certitude), redéfinir ses investissements narcissiques et rendre conflictuelle sa relation à l'objet...». C'est ainsi que l'auteur parvient à discerner entre ce qui caractériserait la certitude par conviction de celle qui prend source dans la croyance. «La certitude, écrit-il, est un tyran qui tue» (De Azambuja, 2004,151). Dans certaines crises que nous traversons et qui ont la saveur de la résurgence mélancolique qui est frappée du sceau d'une disparition, la psychanalyse viendrait-elle emprunter la face cachée de notre propre tyran?

Tout thérapeute individuel est peut-être déjà entrain de disparaître quand il se reconnaît lui-même dans l'attaque en série, dans la sérialisation tournée contre lui que prendrait comme cible, par exemple, un «livre noir de LA psychanalyse». LA psychanalyse, réunifiée et informe pour être mieux visée, n'est peut-être pas celle que l'on porte, individuellement, chacun en-dedans de soi. Mais qu'en est-il du thérapeute, entièrement absorbé à se défendre et à protéger un terrain qu'il reconnaîtrait sien, comme une terre natale à partager mais qui ne lui garantirait pas l'usage de sa langue maternelle: peut-il se limiter à vivre l'attaque que comme si elle se situait au plan individuel? opte-t-il pour une déresponsabilisation en ne situant l'attaque qu'au plan social ? De fait, il risque fort de se retrouver à la fois unique et lié, obligé de surplomber une situation sociale tout en sachant en faire partie.

C'est bien là qu'il devient difficile de trancher et parfois, de s'y retrouver. Face à un éprouvé d'invasion, aux prises avec l'implant étranger qui vient nous tenailler de l'intérieur, nous pouvons être tentés d'accuser la culture ambiante dans laquelle nous baignons. Mais en même temps, nous nous tournons à l'intérieur de nousmêmes pour y chercher la source du malaise et c'est précisément dans ce lieu, à la frontière du dedans et du dehors, que le malaise dans la culture n'arrive plus à occuper une seule des scènes possibles.

\section{Sur fond de scène partagée}

Si la pratique psychanalytique est parfois étrangère à la mode actuelle du rendement efficient et de la productivité à tout prix, si elle ne se sent pas à sa place dans le monde des biens pensants et des vertueux de l'hygiène mentale, on est tenté de se rassurer en pensant qu'elle n'en est pas moins bâtie sur des fondements qui sont tout à fait compatibles avec le réel souci pour l'autre. Quant à sa propre éthique, la psychanalyse parvenait jusqu'à récemment à s'articuler avec les déontologies professionnelles concernées, généralement sans problème. Or, on pourrait toujours débattre à savoir si la pratique psychanalytique est ou non une profession, il demeure que pour plusieurs des praticiens, l'entrée de l'éventuel patient se fera par le biais d'une autre profession.

Là où des expériences d'enquêtes ayant recreusé des traces plus profondes de «fouilles» me laissaient amèrement perplexe, là où le déferlement d'expertises venant désavouer mon travail me jetaient dans l'inquiétude, c'est surtout lorsqu'il m'arrivait d'imaginer la pratique dans un avenir pas si lointain. On pourrait 
reconnaître ici, tout simplement, une réaction courante d'hypervigilance qui consiste à se propulser dans la pensée catastrophique, à tourner vers le futur la difficulté actuelle à composer avec le présent. Mais cela vaut quand même la peine de se questionner jusque dans nos angoisses visionnaires.

$\mathrm{Au}$ Québec, avec la reconnaissance légale du statut de psychothérapeute qui pourrait comporter certains avantages, avec un «contrôle de la qualité » qui serait censé garantir au citoyen l'accès à de meilleurs services, je devenais perplexe devant ces observations plutôt banales : à savoir que «l'être citoyen » se confond de plus en plus avec le «consommateur»; et aussi, à savoir que la culture des «droits» semble de plus en plus faire ombrage à une culture des «devoirs responsables ». Comment le thérapeute psychanalytique allait-il se présenter et se légitimer quand il s'agit, pour lui, de rendre des comptes à l'intérieur d'une logique très puissante qui peut s'énoncer ainsi : avant d'être sujet, l'autre est un citoyen-consommateur, peut-être même une victime potentielle. Allait-on lui demander, à ce thérapeute analytique, d'être plus blanc que blanc, plus articulé dans sa pensée que nul autre sur le marché des thérapeutiques?

Je finissais par me demander, en imaginant ma génération et celles qui suivront: qu'adviendrait-il si la satisfaction des clientèles devenait le critère absolu aux côtés d'un nouvel étalon de réussite qui octroierait à quiconque le droit de ne plus souffrir? Cette question, je me la posais autant en pensant au monde de la psychothérapie qu'à celui de l'éducation. Dans la foulée de mes préoccupations, je pensais alors à mes chers amis, ceux d'un autre métier que le mien et qui sont restés des pédagogues enthousiastes. Mais aussi, ceux qui risquent avec le temps et le cynisme ambiant, de se transformer à leur tour en professeurs de désespoir ${ }^{4} .$. Je pensais donc à ceux de cet autre métier impossible, ceux qui rencontrent chaque jour notre jeunesse en quête de croyances. Deviendraient-ils tentés de n'offrir à ces jeunes, à ces citoyens en devenir, que le droit revendiqué haut et fort à obtenir une note de passage. Mais, de passage vers quoi ? pourrait-on se demander. Ces amis, qui en sont venus à éprouver de la répulsion à l'idée de porter le nom de maîtres, comment pouvait-on leur demander de se soumettre à des plans de réussite constamment soumis aux forces de l'économie et remaniés au gré des fluctuations des prétendus besoins de l'industrie marchande? Qu'en était-il de leur silence, du mien, du nôtre?

Il me semble que le désarroi que l'on éprouve, dans la situation de résistance au traumatique, vient embrasser toutes ces déceptions, tous ces soucis. On devient inquiet, vigilant ou hyper-réaliste et notre pensée s'angoisse des catastrophes dans les lieux mêmes de nos plus chers investissements. L'agglutination, le facteur quantitatif qui édifie sa propre construction, vient bel et bien se nourrir à même la vie et à même sa poussée vers une continuité.

Une des avenues du thérapeute est de se tourner vers d'autres scènes que celle qui lui est familière pour essayer, par le chemin de la différence, de tenter de comprendre mieux dans quel «monde» lui-même vit. Pour ma part, cet épisode trouble fut accompagné d'un besoin de me tremper dans d'autres discours où l'on 
commente et documente, parfois en dénonçant avec force, certains mensonges ou paradoxes de la culture. Croyant me distraire de la réalité psychique, comme si on pouvait facilement y échapper en portant le regard juste un peu à côté, je me trouvai plutôt imbibée par la convergence des discours contemporains sur la chute, sur le déclin, ou encore sur la déroute de la culture actuelle. Je devins momentanément fascinée par diverses théories qui allaient d'une mise en garde à l'autre, en commençant par la dénonciation de l'attaque généralisée contre l'activité de penser et de la manufacturation des idées, en passant par la déconstruction de l'illusion des consentements individuels et par la démonstration des propagandes silencieuses. Enfin, on y étalait le camouflage de la désinformation sous le cumul des informations prétendument hyper-accessibles et on dévoilait les rouages de l'industrie d'un hédonisme radical et sur-valorisé. En bout de ligne, je devenais aussi captivée par les efforts pour décrire la force d'emportement d'un principe technique tournant sur lui-même, sans autre visée que la force motrice de la production en elle-même. Quittant les intellectuels, il me fallut du temps pour saisir jusqu'à quel point j'étais simplement devenue, peu importe le champ d'intérêt, à la fois préoccupée par divers mécanismes relatifs à l'auto-conservation, soucieuse de l'empire du narcissisme, inquiète de toute entreprise qui n'aurait pas de souci pour assurer la reproduction à plus long terme et finalement, inquiétée par ce qui me semblait être un déni massif face à la limite naturelle à l'existence humaine.

Peut-être l'un des avantages de se retremper dans d'autres discours et de chercher à saisir d'autres modes d'analyse de la culture pourrait consister à faciliter un dégagement parfois nécessaire d'avec l'objet sur-investi que deviendrait la psychanalyse lors des luttes que nous avons à mener contre le désaveu. Mais aussi, le trempage dans une autre solution ambiante est susceptible de venir nous rappeler d'autres appartenances qui constituent elles aussi des parties de notre identité sur d'autres scènes de notre vie. Pour ma part, mon incursion dans d'autres champs de l'analyse de la culture m'avait notamment permis de renouer davantage avec ma formation de base qui plaçait la communauté comme objet premier d'étude. C'est par la voix de Charles Taylor, dont le discours philosophique est qualifié de communautariste, que j'entrepris de prendre un certain recul par rapport à la question de l'identité. Lors d'une des occasions de contact avec sa pensée, alors que le philosophe s'adressait à des étudiants, j'avais dressé l'oreille quand j'avais entendu Taylor dire à ces jeunes, en toute simplicité et peut-être comme une mise en garde: «La science excite. Avec elle, on se dit, ça y est... je vais tout savoir!». En quelques secondes, il venait de situer la science en tant qu'objet suprême de séduction qui pourrait exercer une force des plus attrayantes sur le besoin de croire dans un absolu qui semblerait cruellement faire défaut aux cultures profanes.

Puis, j'avais particulièrement été éveillée aussi par sa conception d'un Moi, évidemment fort différent du moi freudien mais qui ne me semblait pas faire ombrage à cette seconde conception, à mesure que divers niveaux qui composent 
l'identité se laissaient saisir en rapport avec la réalité sociale. Il m'avait fallu faire un long détour pour considérer à nouveau que, face aux diverses composantes de notre identité, il fallait bien que chacun de nous ait recours à divers niveaux d'interlocuteurs qui ne peuvent se substituer l'un à l'autre. Taylor, quant à lui, insistait sur une conception de la crise, celle-ci pouvant survenir s'il y a un réel refus de reconnaissance d'une identité ou si une différence devenait trop dérangeante pour être reconnue, enfin si les voies sont réellement bouchées pour faire reconnaître une identité. Dénonçant les risques d'une trop grande marginalité, l'auteur livrait des réflexions éclairantes sur le fait de s'éprouver comme des hors famille ou des hors société, en particulier si la marginalisation atteint un point où l'on ne se sentirait plus dans l'obligation d'agir de façon démocratique ou d'obéir à certaines règles. Peut-être avais-je dû passer par un discours non psychanalytique pour mieux reconnaître certains des enjeux liés à la pratique de la thérapie analytique, une pratique toujours susceptible d'éveiller des crises en chacun de nous et collectivement. L'investissement de cette pratique ne nous place-t-elle pas constamment face l'exigence de survivre, personnellement ou socialement, endehors du bureau comme au-dedans, à l'intérieur même de la rencontre avec ce qu'il y a de plus privé en chaque individu et donc, de ce qu'il y a aussi de plus collectif et indifférencié?

Dans le souffle d'un vent communautaire, il me vint que les amitiés sont peutêtre ce qui viendra offrir au thérapeute l'énergie motrice pour effectuer à la fois un exigeant travail de communication mais aussi, et surtout, pour continuer de l'intérieur le travail d'auto-analyse exigé. Ces amitiés, que l'on pourrait souhaiter comme soutenantes mais non complaisantes, serviront possiblement de relais pour maintenir le repère fondamental qu'a constitué l'analyse personnelle, en nous aidant à maintenir un travail d'éveil et de résistance auquel nous sommes tous constamment convoqués. Par le mot résistance, j’entends non pas le concept auquel la psychanalyse a recours, mais un rappel imaginaire du travail sous-terrain que nous associons souvent à une opposition délibérée et stratégique. Cette résistance est de celles qui nécessitent que nous comprenions davantage les règles du jeu qui ont cours dans la horde contemporaine à laquelle nous appartenons. Cette résistance nécessaire, peut-être est-ce celle qui s'opposerait à la tentation de n'occuper que les places privilégiées de la conformité, de la vertu ou de l'empathie.

\section{D'une réalité à l'autre}

En soi, l'illustration d'épisodes cliniques peut susciter un intérêt pour d'autres cliniciens. Cela est aussi nourri, pour ma part, à la fois d'un désir d'appropriation autant que d'un appel à l'effort collectif de pensée. Ainsi, requérir un interlocuteur (autre que l'interlocuteur interne) fait appel au tiers externe à qui on laisse un pouvoir de dire quelque chose au sujet des efforts de théorisation qu'il nous faut ébaucher en solitaire dans la clinique. Ce que Sophie deMijolla-Mellor (2004) attribue au «théoricien», peut-être devons-nous l'attribuer aussi au «clinicien» 
qui ne cesse de se formuler à lui-même une compréhension des expériences auxquelles il participe. Soulignant l'importance d'un critère extérieur comme mode de validation des enjeux identitaires sous-jacents à nos formations théoriques, elle rappelle que «rien n'assure a priori le théoricien qu'il n'est pas en train de mettre en forme un fantasme privé, aucun signe intérieur et surtout pas la certitude dont il sait qu'elle peut s'attacher à ces "fausses théories", infantiles ou délirantes » (De Mijolla-Mellor, 2004,241). Ce rapport essentiel à autrui n'a-t-il pas une valeur tout aussi primordiale dans toute situation traumatique, quand la charge excessive combinée à l'effet d'enfermement menacent l'appareil psychique de l'individu ?

L'incertitude au sujet du témoignage de nos propres sens et de notre propre capacité à en faire émerger une pensée est une possibilité d'erreur que chacun de nous peut redouter. Tout au long de cet épisode se profilait, avec un certain désarroi, la parole d'un enfant solitaire cherchant à saisir l'énigme des adultes à qui il demanderait timidement: «mais comment font-ils, les autres, pour paraître si rassurés, si insouciants? comment arrivent-ils à simplement jouer?». Le reste toujours actif, jamais complètement liquidé, des questions infantiles pour lesquelles les représentations et les mots ont pu nous faire défaut ne sont-ils pas le terrain le plus fertile à nos questionnements ultérieurs? Or, tout effort pour comprendre «Je» ou «le monde», ou l'un en rapport avec l'autre, n'est-il pas lié avec ce besoin de refonder un peu plus solidement nos propres origines et l'origine de notre propre pensée ? N'est-ce pas aussi à cela que nous sommes constamment convoqués?

L'épisode illustré ici est venu rappeler que lorsqu'il s'agit pour la personne du thérapeute, dans toute son individualité et sa solitude, de se situer face à l'ensemble de sa pratique, divers petits faits anodins et courants viendront le détourner de maintes façons de ce qu'il tient généralement comme une évidence, à savoir la préséance de l'inconscient dans les situations impliquant des humains avec d'autres humains. Or, l'analyse des événements en tant que faits psychiques s'offrira au thérapeute afin qu'il préserve ses outils de travail tels : sa patience, sa contenance, ses renoncements, sa capacité à être en lien, son plaisir et parfois sa douleur à penser et à théoriser et peut-être enfin pourrions-nous ajouter sa dignité à exercer son métier.

En particulier devant la tentation sociologisante à laquelle il est exposé en maintes occasions, l'exploration individuelle par le thérapeute des éléments se rattachant à des faits psychiques sociaux, qui empruntent aux logiques de la horde et à celle du père (Imbeault, 2004), ouvriront peut-être la voie à son dégagement psychique personnel. Ces faits psychiques, qui peuvent se superposer et s'entremêler à sa perception de la réalité sociale, se présenteront comme des obstacles alors qu'ils pourraient servir de leviers à l'élaboration.

Par ailleurs, les situations à forte teneur d'ébranlement du moi rencontrées dans la pratique, si ce n'est que par leur valence quantitative, forceront le thérapeute à un travail intensif pour d'abord permettre son jugement de réalité. Or, si ce jugement doit tenir compte de la tendance du moi à s'aliéner au monde extérieur 
pour justifier le recours à ses défenses, il ne pourrait se passer de la reconnaissance essentielle concernant la nature même de la réalité psychique qui prendrait son essor à même une recomposition active d'objets internes (Scarfone, 2007). Ainsi, autant le thérapeute sera mis au défi de requalifier la nature particulière de la réalité psychique qui procède selon sa propre logique, autant il sera mis au défi de tenir compte des différentes logiques qui animent la scène de l'infantile sur laquelle l'individu ne cesse de reconstruire.

L'imbrication de diverses logiques auxquelles nous avons emprunté dans cet épisode clinique (que ce soit par le recours à des logiques maternelle et paternelle, logiques de fonction et de statut, logiques individuelle et collective) est venue suggérer le risque réel de fouillis quand diverses logiques tentent de cohabiter et de se faire entendre. Ce fouillis peut être tel que même les désaccords pourraient ne plus y trouver leur place, encore moins leur entendement. Partant de diverses logiques auxquelles nous sommes confrontés dans les multiples discours de la culture et dans les diverses expériences qu'elle nous impose, il reste à chaque thérapeute de poser plus clairement ce qui le met personnellement au défi dans sa capacité de rester: à la fois un héritier d'une culture de la pensée et à la fois un héritier d'une culture des soins thérapeutiques.

Sur la scène de la horde contemporaine dont il fait partie, le thérapeute est appelé à discerner des degrés d'un déni dans lequel il baigne. Il lui faut tenir compte de la force de rejet, d'attaque ou de désaveu auquel il croit parfois faire face. Ce «degré » a son importance et par le biais d'un des mots d'ordre qui nous est souvent lancé avec vigueur («soyez donc réalistes!») s'énonce un refus de dialogue qui feint la bonne volonté. Or, l'injonction au réalisme, qui ne tient pas compte de l'ensemble de la réalité humaine, appartient bel et bien à l'une des logiques propres à notre culture actuelle, elle vient même en recouvrir les plus grandes illusions. Quant à l'hypothèse de l'inconscient, comment la maintenir autravers ce discours afin qu'elle puisse toujours valoir la peine d'être vérifiée ? Comment en conserver l'attrait afin qu'elle conserve un pouvoir de séduction, propre à fonder un transfert de base suffisant sur lequel viendra s'étayer la rencontre réelle entre deux humains?

Afin que l'inconscient puisse conserver son statut de fait, un fait propre à la réalité psychique humaine, la présence de témoins diversifiés paraît plus que requise. Ces témoins sont convoqués pour tenir parole, pour continuer à tenir la place de la parole et cela, sur d'autres terrains aussi que celui de la psychanalyse. Peut-être est-ce là que se loge un des espoirs que Natahlie Zaltzman traduisait déjà il y a dix ans en situant la culture et la raison comme ce qui «tient tête à la volonté de mort dans l'humain» (Zaltzman, 1998,67). Tenir tête à la volonté de mort n'est sûrement pas du même registre qu'effectuer des résurrections, toute la nuance est là. Or, sur le marché des thérapeutiques, la demande de production de résurrections ou d'autres miracles est toujours là, nourrissant la disposition à la croyance et à la magie que tous les enfants connaissent, que cela les renvoie ou non, devenus adultes, à des étendards politiques, religieux, thérapeutiques, hygiénistes ou autres. 
La culture actuelle semble sécréter un incroyable gouffre à penser. La paresse, la quête d'un plaisir immédiat, tout autant que le désir de facilité comptent parmi ce qui pourrait nous renvoyer, individuellement ou collectivement, à l'illusion d'une espèce de confort masturbatoire, intellectuel ou autre, que l'on justifierait par un impossible partage. Peut-être sous-estimerait-on alors les risques inhérents à l'idée d'incommunicabilité. Ainsi, les restes du désir de tenir l'objet sous notre emprise, de ne pas risquer de le perdre ni de l'abîmer (cela inclut l'objet de la psychanalyse et la psychanalyse en tant qu'objet), cela nous confronte à bien des renoncements qui sont pourtant nécessaires si l'on souhaite réellement s'inscrire dans une logique de vie. Cette logique de vie requiert de chacun de nous, même devenus adultes, de s'extraire à un certain enfermement qui ne saurait se confondre avec ce que certains ont déjà nommé une splendide solitude.

marie desrosiers

583 rue ladouceur

joliette (québec)

j6e $3 \mathrm{w} 4$

desrosiersmarie@ videotron.ca

\section{Notes}

1. À la relecture de quelques chapitres de ce livre, il me serait difficile de localiser, de ces récits qui font voir l'analyste au travail, ce qui m' aurait laissé tout particulièrement cette impression. Cependant, Granoff y témoigne de maintes façons d'un «désir d'analyse» qui s'empare de la situation analytique, avec les risques d'en oublier des singularités en jeu.

2. Je fais ici référence à une idée possiblement secondaire (si l'on se réfère à la densité de l'article en question) qui m'apparaît à la lecture de cette conférence prononcée en 2002: «Y a-t-il un au-delà de la cruauté pour la psychanalyse ? Ou La raison de la raison à venir».

3. Il s'agirait en fait de l'énoncé d'un autre qui est repris par Pontalis (1994) à l'occasion de son entretien avec la revue Le Débat.

4. En rappel de l'ouvrage de Nancy Huston (2004).

\section{Références}

DeAzambuja, M., 2004, L'amour des certitudes, Penser/Rêver, Mercure de France, n ${ }^{\circ}$, Des érotomanes, 149-163.

DeMijolla-Mellor, S., 2004, Le besoin de croire. Métapsychologie du fait religieux. Paris, Dunod.

Eliacheff, C., Soulez Larivière, D., 2007, Le temps des victimes, Paris, Albin Michel.

Ferenczi, S., 1929, Euvres complètes, Psychanalyse IV, Paris, Sciences de l'homme Payot, 1982.

Granoff, W., 2004, Le désir d'analyse, France, Édition Flammarion/Aubier.

Gribinski, M., 2007, La chose déchirée, in La psychanalyse révisée, France, Éditions de l'Olivier, Collection Penser/Rêver, 7-10.

Huston, N., 2004, Professeurs de désespoir, France, Actes Sud, collection Babel.

Imbeault, J., 2004, «Le père n'aime que moi », Penser/Rêver, Mercure de France, nº 5, Des érotomanes, 165-178. 
Laperrière, R., 1999, Le malaise de l'imposteur, Filigrane, vol.8, nº 2, 88-99.

Major, R., 2003, La démocratie en cruauté, Paris, Édition Galilée.

Pontalis, J.B., 1994, Détournement de psychanalyse ?, Le Débat, numéro 70, mars-avril.

Scarfone, D., 2007, «Seul ce qui est humain peut nous être étranger», in Fédida, P. et. al., Humain/Déshumain, Paris, Presses Universitaires de France, 217-237.

Zaltzman, N.,1998, De la guérison psychanalytique, Paris, Presses universitaires de France. 